\title{
Compliance of Hour-1 Sepsis Care Bundle in Patients with Surgical Infection Requiring Surgery in Hospital Tengku Ampuan Afzan
}

Dhayalan Ganisan, Jiffre Din

Department of General Surgery, Hospital Tengku Ampuan Afzan, Pahang Darul Makmur, Malaysia

Presenter: Dhayalan Ganisan

Introduction: Sepsis is life-threatening organ dysfunction caused by a dysregulated host response to infection. According to Sepsis 3 definition, an increase of 2 or more in the qSOFA score should create a suspicion of sepsis and organ dysfunction. It is a dire condition as studies show; patients with a qSOFA score of $\geq 2$ have a mortality rate of $\geq 0 \%$. The Surviving Sepsis Campaign guidelines have depicted an Hour-1 Sepsis Bundle in managing patients with sepsis which has proven to reduce morbidity and mortality. The objective of this study was to analyze the demographic data of patients presenting with surgical sepsis requiring surgery and compliance to the Hour-1 Sepsis Bundle, concurrently the mortality rate of these patients. Materials and Method: A retrospective epidemiological study was done from the $1^{\text {st }}$ of October to the $31^{\text {st }}$ of December 2018. Patients included in the study were all patients with evidence of surgical infection requiring emergency surgery and patients presenting with trauma were excluded. This number of patients was then scrutinized into patients with evidence of sepsis, subsequently the compliance to the Hour-1 Sepsis Bundle. Results: The analysis showed that 23 of the 156 patients with surgical infection requiring surgery were in sepsis. Of this, 5 patients succumbed during the same admission. Results also showed patients who developed sepsis in their $5^{\text {th }}$ decade of life are more susceptible to death. In accordance with the Hour-1 Sepsis Bundle, compliance to measuring lactate level was $70 \%$, obtaining blood culture was $47.8 \%$, administration of antibiotics was $82.6 \%$ and rapid administration of crystalloid was $0 \%$. All three patients requiring vasopressors were started on vasopressors within the hour. Conclusion: Compliance to the Hour-1 Sepsis Bundle in whole was unsatisfactory. This was noticeably apparent especially to fluid resuscitation where it was not delivered as recommended by the Sepsis Bundle. 